\title{
Lossy mode resonances in photonic crystal fibers
}

\author{
Mauricio Salazar Sicacha', Vladimir P. Minkovich ${ }^{1 *}$, Alexander B. Sotsky², Artur V. Shilov², Luidmila I. Sotskaya ${ }^{3}$ and \\ Evgeny A. Chudakov²
}

\begin{abstract}
The interaction effect of the fundamental mode in a special photonic crystal fiber (PCF) with a thin-film absorbing coating deposited on a surface of a fiber cladding on the optical transmission of the PCF is theoretically studied. It is shown that the transmission has a multi-peak spectrum that is determined by the resonance capture of the fundamental PCF mode energy by the coating. In some cases, this capture is explained by a resonance coupling between the fundamental core mode and leaky modes of the coating, or between the fundamental PCF mode and cladding modes located between PCF air channels and the coating. Examples are presented of using this effect to develop fiber-optic sensors of refractive index or pressure, and to sense a nanoscale adsorption layer of ammonia molecules deposited on a coating surface contacting air.
\end{abstract}

Keywords: Optical fiber devices, Optical fiber sensors, Optical fiber taper, Photonic crystal fibers, Lossy mode resonance

\section{Introduction}

Fiber-optic physical and chemical sensors using lossy mode resonance (LMR) have been intensively investigated $[1,2]$. The LMR occurs due to the interaction of the light propagating through an optical fiber coated with an absorbing film of appropriate optical properties. If coating modes are in phase matching with fiber modes, then the fiber modes may couple to the coating modes, generating a lossy mode. LMR will suffer a variation if either a variation in the properties of the LMR support film (either refractive index or thickness) or changes in the optical properties of the surrounding media occur. These variations result in a detectable modulation of the fiber transmission spectrum, which is a sensory effect.

In most cases, such devices use standard optical fibers, in which mode propagation constants exceed the refractive index of the cladding. Then the phase matched

\footnotetext{
* Correspondence: vladimir@cio.mx

'Division of Photonics, Centro de Investigaciones en Optica, 37150 León, Mexico

Full list of author information is available at the end of the article
}

modes of the fiber and the film have evanescent fields in the fiber cladding. The coupling of these modes implies a close contact between the fiber core and the absorbing film. It is achieved by removing the cladding (or a part of it) in a segment of the fiber and using a different coating material to substitute for it $[1,2]$.

In this paper, we theoretically consider another possibility of realizing the LMR, which is provided by the use of a special photonic crystal fiber (PCF) [3]. If PCF is formed by a finite number of air channels in a dielectric matrix, then propagation constants of PCF modes are usually less than the refractive index of the matrix $[3,4]$. As a result, if the matrix is unbounded, then fields of these modes leak from the fiber core, that is, the modes suffer confinement losses [3, 4]. In real PCFs with bounded claddings and absorbing film coatings, such leaky modes can be phase matched only with the leaky ones of the film coatings. The coupling of leaky modes of different waveguides may take place at a significant distance between the waveguides. This makes it possible to obtain the LMR due to the coupling between modes of a PCF core and modes of an absorbing film coating 
applied directly to the outer surface of the fiber cladding without a local modification of the cladding [5]. Since at the current level of technology, PCFs can be easily fabricated by single-mode ones [3], the described LMR has the advantages of enabling the single-mode operation of sensors and leading to the almost complete elimination of diffraction losses at irregularities of the PCF.

The LMR in PCFs was first investigated in [5], where the interpretation of the experimentally observed quasiperiodic transmission spectrum of a regular PCF with an absorbing polymer coating was given. The purpose of this work is to assess features for using the LMR in PCFs for sensing applications. Numerical estimates are presented both for modal properties of PCFs with absorbing coatings and for transmission spectra of PCFs in sensing refractive index of a liquid, pressure in the liquid and an adsorption nanoscale layer. The modeling is performed with the use of the method of integral equations [5], which is the full vectorial mode solver. It is formulated with respect to the transverse components of the magnetic field within a PCF, and makes it possible to perform a rigorous calculation of the fields and propagation constants of guided and leaky modes of PCFs with layered coatings.

\section{LMR in PCFs}

To study regular and adiabatically tapered PCFs with absorbing coatings, calculations are performed for the time factor exp. $(i \omega t)$. The special PCF used in our calculations consists of a solid core surrounded by three rings of air channels arranged in a hexagonal pattern. The PCFs material is quartz glass. Its refractive index $n_{s}$ is supposed to be real and described by the well-known three-term Sellmeier formula [6]. Parameters of the untapered PCF are following: cladding diameter $D_{0}=$ $123.4 \mu \mathrm{m}$, diameter of the air holes $a_{0}=3 \mu \mathrm{m}$, photonic crystal pitch $\Lambda_{0}=8.8 \mu \mathrm{m}$ [7]. The structure of the adiabatically tapered PCF is presented in Fig. 1. At taper fabrication, the diameter of holes $a$ and the pitch $\Lambda$ within the taper waist are modified according to the rule [8] $a=\left(2 A_{w} / D_{0}\right) a_{0}, \Lambda=\left(2 A_{w} / D_{0}\right) \Lambda_{0}$. For taper calculations, we used $2 A_{w}=44 \mu \mathrm{m}$ and $L=3 \mathrm{~cm}$ as values (see Fig. 1); the selected values for the parameters correspond to single-mode PCFs [3, 7]. Therefore, in what follows, the PCF modes are understood as the PCF fundamental modes.

We will consider two types of PCF modes: modes of the first type are leaky modes of PCFs with an infinite cladding, and we will call them PCF core modes, whereas modes of the second type are modes of a PCF with a bounded cladding and an absorbing coating, and we will simply call them PCF modes. Modes of the first and second types have different imaginary parts of the propagation constants, but almost coinciding real parts of the propagation constants and transverse field distributions within the PCF core [5].

Figure 2 refers to the untapered PCF $\left(2 A_{w}=D_{0}\right)$. It illustrates modal dispersion $\beta(\lambda)$, where $\beta$ are dimensionless complex propagation constants of PCF modes of both the above types and modes of the absorbing film coating, and $\lambda$ is the light wavelength. The PCF is supposed to be coated with multifunctional butyl acrylate, having a complex refractive index $n_{c}=1.54$ i0.00002 (below that is the polymer coating), and surrounded by water $\left(\varepsilon_{w}=n_{w}^{2}, n_{w}=1.33\right.$, see Fig. 1$)$. 'Loss' in Fig. 2a means $4 \pi 10^{7}|\operatorname{Im} \beta| /(\lambda \ln 10)$, where $\operatorname{Im} \beta$ is the imaginary part of the propagation constant of the PCF mode.

Figure $2 \mathrm{~b}$ shows spectral dependences of the refractive index for quartz glass, the complex propagation constants of the PCF core mode and the modes of the absorbing coating. It allows one to qualitatively explain the non-monotonic character of the dependence shown in Fig. 2a.

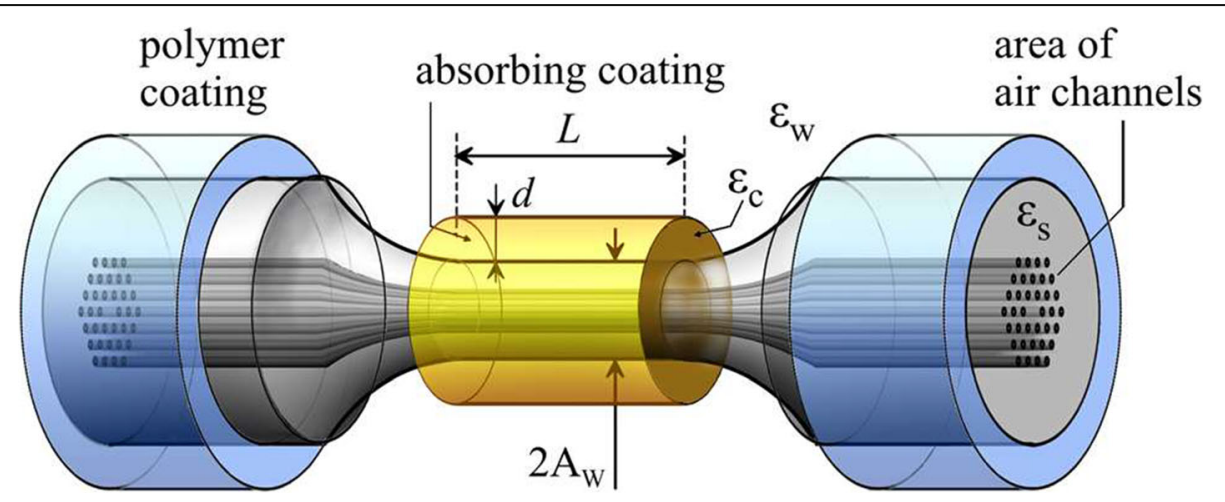

Fig. 1 An adiabatic taper of the PCF with three hexagonal rings of air channels and an absorbing coating of length $L$ and thickness $d$ around the taper waist of diameter $2 A_{w}$ 

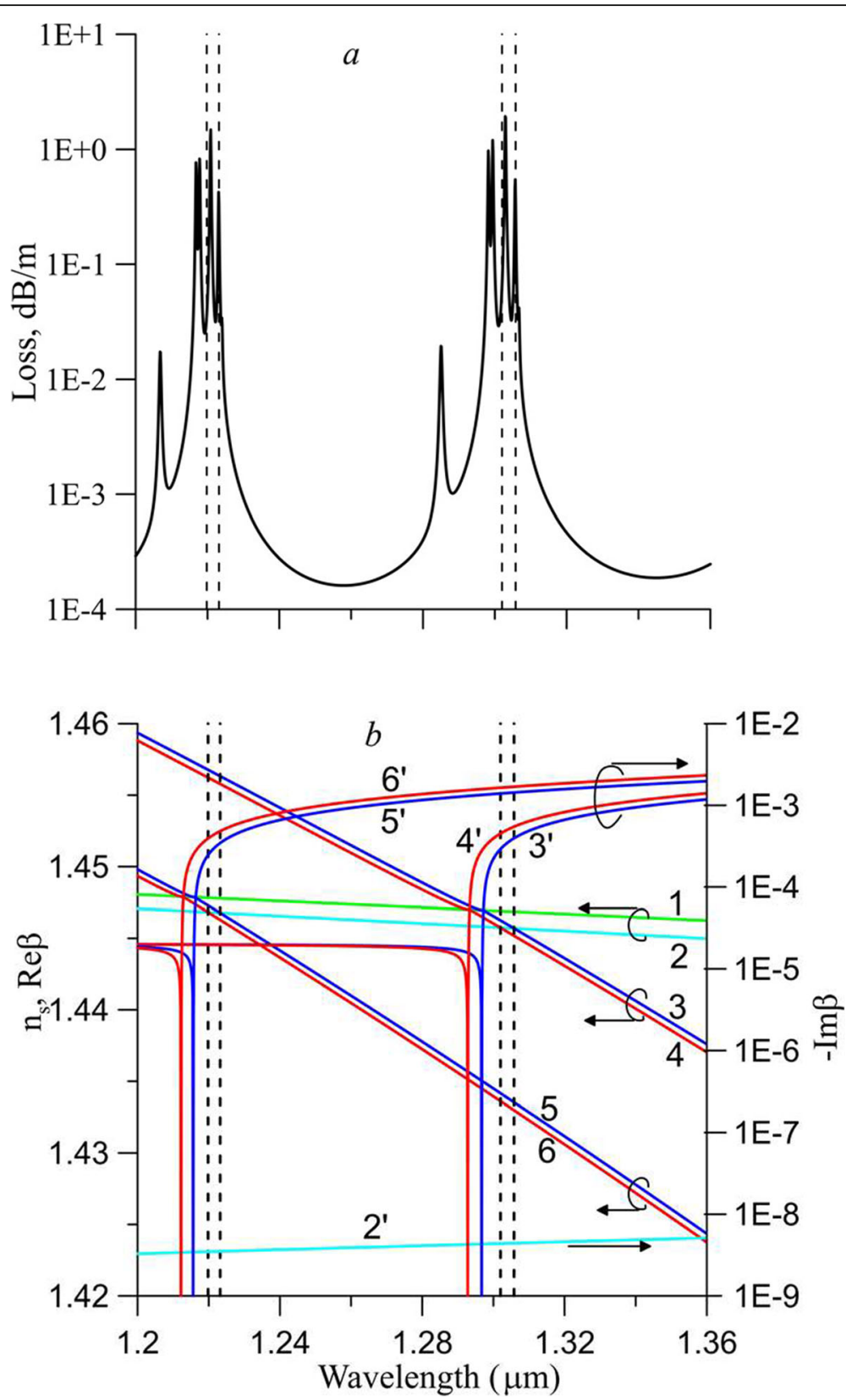

Fig. 2 Spectral dependencies of mode propagation constants for an untapered PCF with a polymer coating of thickness $d=20 \mu m$, surrounded by water; $\mathbf{a}$-loss of the PCF mode, $\mathbf{b}$-phase diagram. Curve 1 - quartz glass refractive index, 2 and $2^{\prime}-\operatorname{Re} \beta$ and $\operatorname{Im} \beta$ of the PCF core mode, 3 and $3^{\prime}, 5$ and $5^{\prime}-\operatorname{Re} \beta$ and $\operatorname{Im} \beta$ of TE modes of different orders for the coating, 4 and 4', 6 and $6^{\prime}-\operatorname{Re} \beta$ and $\operatorname{Im} \beta$ of TM modes of the same orders for the coating. Dashed lines indicate wavelengths of phase synchronism of the PCF core and coating modes. They correspond to intersection points of curves 3, 4, 5 and 6 with curve 2

The PCF core mode was calculated on the assumption that $\varepsilon_{w}=\varepsilon_{c}=\varepsilon_{s}=n_{s}^{2}$. According to Fig. $2 \mathrm{~b}$, for this mode, $\operatorname{Re} \beta<n_{\mathrm{s}}$; that is, it leaks from the PCF core. At the same time, as $\operatorname{Re} \beta>n_{\mathrm{w}}$, the PCF mode field experiences total internal reflection at the outer boundary of the PCF coating. Therefore, and also since $\operatorname{Im} n_{s}=0$, loss of the PCF mode in Fig. $2 a$ is entirely caused by dissipation of its energy in the coating. The corresponding solid curve in Fig. $2 \mathrm{a}$ and curves 2, 2' in Fig. 2b are computed through the method of integral equations [5].

Curves $3-6$ and $3^{\prime}-6^{\prime}$ in Fig. $2 b$ are the results of a numerical solution by means of contour integration method [9] of the dispersion equation for modes of a planar dielectric waveguide [10]. 


$$
\begin{aligned}
& {\left[\frac{\gamma_{w}}{\sigma}\left(\frac{\varepsilon_{c}}{\varepsilon_{w}}\right)^{T}-\frac{\sigma}{i \gamma_{s}}\left(\frac{\varepsilon_{s}}{\varepsilon_{c}}\right)^{T}\right] \sin \left(k_{0} \sigma d\right)} \\
& \quad+\left[1+\frac{\sigma}{i \gamma_{s}}\left(\frac{\varepsilon_{s}}{\varepsilon_{c}}\right)^{T}\right] \cos \left(\sigma k_{0} d\right) \\
& \quad=0
\end{aligned}
$$

where $T=0$ for TE modes, $T=1$ for TM modes, $\gamma_{w}$ $=\sqrt{\beta^{2}-\varepsilon_{w}}\left(\operatorname{Re} \gamma_{w} \geq 0\right), \gamma_{s}=\sqrt{\varepsilon_{s}-\beta^{2}}\left(\varepsilon_{s}=n_{s}^{2}\right.$,

$$
\left.\operatorname{Re} \gamma_{w} \geq 0\right), \sigma=\sqrt{\varepsilon_{c}-\beta^{2}}\left(\varepsilon_{c}=n_{c}^{2}, \operatorname{Re} \sigma \geq 0\right), k_{0}=2 \pi / \lambda,
$$

modeling the absorbing coating of thickness $d$. These curves are computed based on the reasons that, from the point of view of the ray optics, the fundamental mode of the PCF is mainly formed by meridional rays [5]. When considering a reflection of such rays from the coating, the latter can be approximately replaced by a planeparallel layer of thickness $d$. In this approximation, the coating modes should be understood as the modes of the planar dielectric waveguide. Due to hybrid polarization of the PCF core mode, it can be coupled with both TE and TM modes of the coating [5].

In Fig. 2b, segments of curves 3-6 located above curve 1 correspond to guided modes of the planar dielectric waveguide, and those below curve 1 correspond to leaky modes of the waveguide. The transition in the mode type from guided to leaky occurs as the wavelength increases [10]. At the moment of this transition at the critical wavelength $\lambda=\lambda_{c}$, the mode propagation constant $\beta$ coincides with the refractive index of the quartz glass, $\mathrm{n}_{\mathrm{s}}$. In this case $-\operatorname{Im} \beta$ reaches its minimum. As the difference $\lambda-\lambda_{c}$ grows, the value of $-\operatorname{Im} \beta$ increases sharply due to the leakage effect (see Fig. 2b).

At the same time, curve 2 passes everywhere below curve 1 in Fig. 2b; so, the PCF core mode is always leaky. As a result, this mode can be phase matched with only leaky modes of the coating at resonance wavelengths indicated in Fig. 2 by vertical dashed lines (at these wavelengths, real parts of the propagation constants of the modes coincide); in Fig. 2a, the loss of the PCF mode sharply grows in the vicinity of these wavelengths. As observed in Fig. 2a, a quasi-periodic spectrum of the PCF mode loss can be explained by the resonance coupling of the PCF core mode with leaky modes of the absorbing coating [5]. This coupling leads to a resonance capture of the PCF mode energy by the coating, and to the release of heat in it.

It should be noted that there is a large difference of about 5 orders of magnitude between the imaginary parts of the propagation constants of the PCF core mode and the absorbing coating modes in the vicinity of the resonance wavelength in Fig. $2 \mathrm{~b}$. This mismatch of propagation constants negatively affects the coupling noted. Since the attenuation of the coating modes sharply decreases with decreasing wavelength (see Fig. $2 b)$, it causes a left shift of the centers of gravity of the attenuation peaks relative to the dashed lines in Fig. 2a. This shift increases with an increase in attenuation of the coating modes (see below).

Note also that the simple qualitative coupling model described does not allow us to fully explain the complex multi-peak loss dependence $(\lambda)$ in Fig. 2a.

When using untapered PCFs, the mentioned coupling of leaky modes can be rather weak. As a result, it can only be observed when sufficiently long pieces (of the order of meters) of coated untapered PCFs are used (this conclusion follows from Fig. 2a). Besides, to realize the LMR, it is necessary to keep the coating thickness $d$ constant along the entire sensitive piece of the PCF, with a tolerance of $0.075 \lambda / \sqrt{n_{c}^{2}-n_{s}^{2}}$ [5]. For long PCF pieces and thick coatings, this requirement is difficult enough to fulfill. It is suggested that the use of thinner coatings (less than $20 \mu \mathrm{m}$ ) and adiabatic PCF tapers with a length of a sensitive region (taper waist) of the order of centimeters will overcome these difficulties.

Modal properties of untapered and tapered PCFs with the coating of the same polymer but with a thickness of $5 \mu \mathrm{m}$ are shown in Figs. 3 and 4. These Figs. have the same sense as Fig. 2, but with decreasing $d$, the spectral intervals between the dispersion curves of the coating modes are increased [5]. As a result, in the spectral range of Figs. 3 and 4, there are only two (one TE and one TM) coating modes that can be resonance coupled with the PCF core modes.

In Fig. 3, resonance wavelengths are located in the vicinity of $\lambda=1.24 \mu \mathrm{m}$. As can be seen from Fig. 3a, the peak attenuation of the untapered PCF mode remains comparable with the one in Fig. 2a. This means that it is determined mainly by the confinement losses of the PCF core mode, which are the same in both cases. But in the vicinity of the resonance wavelengths values of $-\operatorname{Im} \beta$ in Fig. 3b, they are approximately 3 times greater than the analogous values in Fig. $2 \mathrm{~b}$. Thus, the decrease in the coating thickness increases the mismatch of the complex propagation constants of the PCF core mode and coating modes. It causes a more pronounced left shift of the centers of gravity of the attenuation peaks relative to the dashed lines in Fig. 3a in comparison with the analogous shift in Fig. 2a. To explain this shift quantitatively, we have calculated the wavelengths that correspond to the minima of the moduli $\left|\beta-\beta_{\mathrm{TE}}\right|$ and $\left|\beta-\beta_{\mathrm{TM}}\right|$, using the phase diagram presented in Fig. $3 \mathrm{~b}$. Here $\beta, \beta_{\mathrm{TE}}$ and $\beta_{\mathrm{TM}}$ are dimensionless complex propagation constants of the PCF core mode, TE and TM mode of the coating, respectively. These wavelengths are indicated in Fig. 3a by dotted lines (the left of the lines 

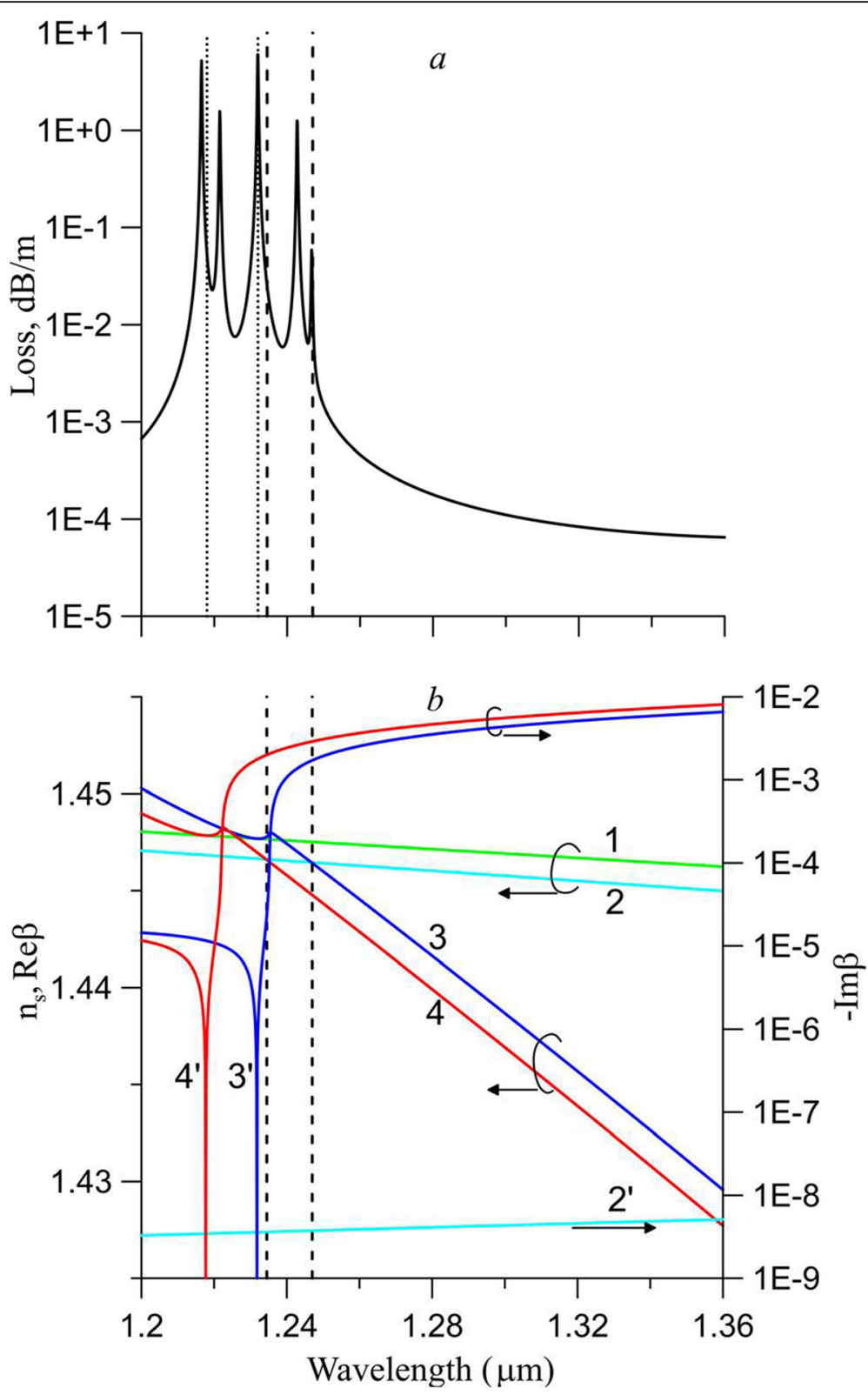

Fig. 3 Spectral dependencies of mode propagation constants for an untapered PCF with a polymer coating of thickness $d=5 \mu m$ surrounded by water; $\mathbf{a}$-loss of the PCF mode, $\mathbf{b}$-phase diagram. Curve 1 - quartz glass refractive index, 2 and $2^{\prime}-\operatorname{Re} \beta$ and $\operatorname{Im} \beta$ of the PCF core mode, 3 and 3' - Re $\beta$ and $\operatorname{Im} \beta$ of TE mode of the coating, 4 and $4^{\prime}-\operatorname{Re} \beta$ and $\operatorname{Im} \beta$ of TM mode of the coating. Dashed lines indicate wavelengths of phase synchronism of the PCF core and coating modes. They correspond to intersection points of curves 3 and 4 with curve 2. Dotted lines refer to minima of $\left|\beta-\beta_{T M}\right|$ and $\left|\beta-\beta_{T E}\right|$

refers to min $\left|\beta-\beta_{\mathrm{TM}}\right|$, right - to $\left.\min \left|\beta-\beta_{\mathrm{TE}}\right|\right)$. As can be seen, they are correlated well with the main loss maxima.

To enhance the coupling of the PCF core and coating modes and, respectively, the attenuation of the PCF mode, it is possible to use an adiabatic PCF taper (see Fig. 4). According to Figs. $3 \mathrm{a}$ and $4 \mathrm{a}$, such a device allows a more pronounced influence of the absorbing coating on PCF transmission. In particular, in Fig. 4a the maximum of the Loss dependency $(\lambda)$ is an order of magnitude greater than the analogous maximum in Fig. $3 a$, which allows a decrease of an order of magnitude in the length of the fiber segment with the LMR; however, in accordance with Figs. $3 \mathrm{~b}$ and $4 \mathrm{~b}$, the transition to tapered PCF leads to a decrease in $\operatorname{Re} \beta$ and an increase in $-\operatorname{Im} \beta$ of the PCF core mode, leading to a further mismatch of the complex propagation constants of the PCF core mode and coating modes. As a result, the above 

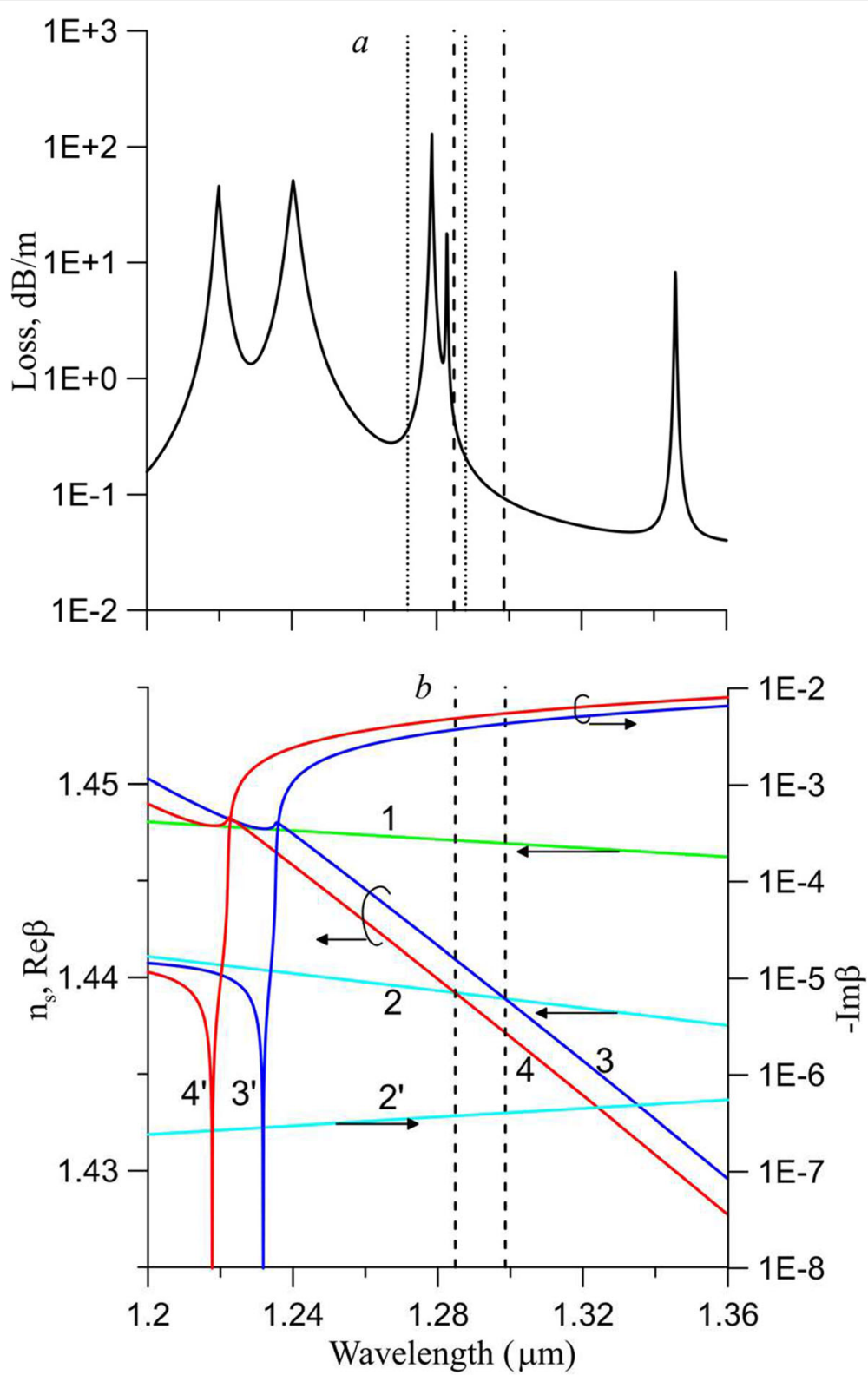

Fig. 4 Spectral dependencies of mode propagation constants for the tapered PCF with a polymer coating of thickness $d=5 \mu m$ surrounded with water; $\mathbf{a}$-loss of the PCF mode, $\mathbf{b}$-phase diagram. Curve 1 - quartz glass refractive index, 2 and $2^{\prime}-\operatorname{Re} \beta$ and $\operatorname{Im} \beta$ of the PCF core mode, 3 and $3^{\prime}$ - Re $\beta$ and $\operatorname{Im} \beta$ of TE mode of the coating, 4 and $4^{\prime}-\operatorname{Re} \beta$ and $\operatorname{Im} \beta$ of TM mode of the coating. Dashed lines indicate wavelengths of phase synchronism of the PCF core and coating modes. They correspond to intersection points of curves 3 and 4 with curve 2. Dotted lines refer to minima of $\mathbb{B}-\beta_{\text {TM }}$ (left line) and $\mathbb{B}-\beta_{\text {TE }}$ (right line)

mentioned simple coupled mode model cannot completely explain transmission properties of the adiabatically tapered PCF. Indeed, in Fig. 4a two sharp loss peaks located in the vicinity of resonant wavelengths $\lambda=$ $1.285 \mu \mathrm{m}$ and $\lambda=1.299$ are significantly shifted relative to these wavelengths. Dotted lines in Fig. $4 a$, which have the same meaning as in Fig. 3a, better match with these peaks, but they also are not aligned with the peaks in detail. Besides, along with the marked peaks a number of other broader attenuation peaks are observed. These additional peaks can be attributed to a resonance coupling of the PCF core mode with modes of the PCF cladding that are concentrated in the area between air channels and the absorbing coating [8], and therefore, effectively interact with the coating.

Figure $5 \mathrm{a}$ and $\mathrm{b}$ refer to the loss maxima observed at wavelength $\lambda=1.244 \mu \mathrm{m}$, which is far from the pair of dashed lines in Fig. 4, and at wavelength $\lambda=1.279 \mu \mathrm{m}$; 


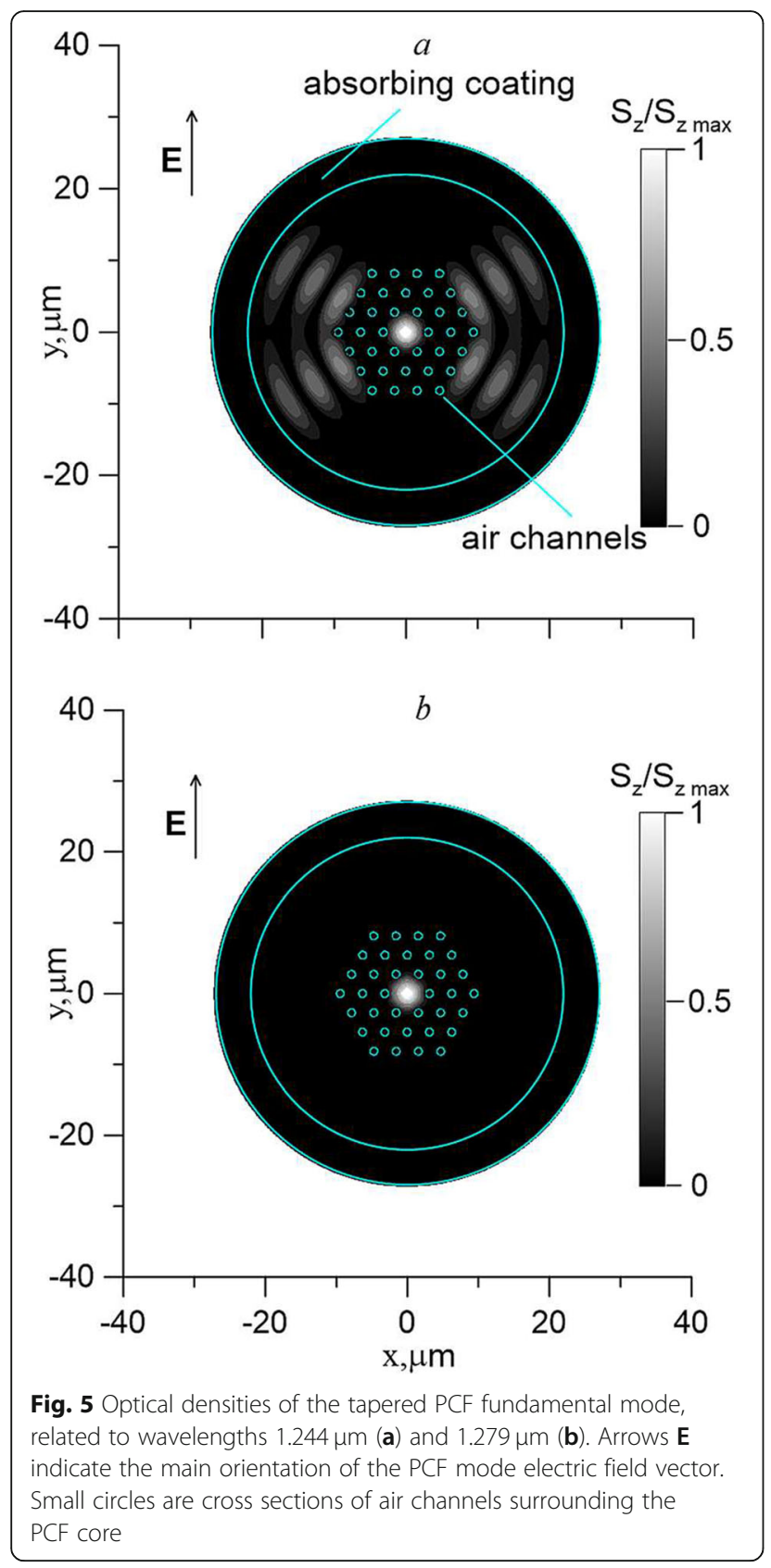

that can be attributed to the direct coupling between the tapered PCF mode and modes of the absorbing coating (see Fig. 4).

This mechanism is confirmed by considering Figs. 5 and 6 , which compares the normalized intensity distributions $S_{z}(x, y) / S_{z \max }$ for the tapered PCF mode, related to different wavelengths. Here $S_{z}(x, y)$ is the z-component of the Poynting vector of the PCF mode field (axis $\mathrm{OZ}$ is directed along the PCF).

It should be noted that on the linear scale of $S_{z}(x, y) / S_{z}$ $\max$ representation used in Fig. 5, with the exception of a narrow vicinity of wavelength $\lambda=1.244 \mu \mathrm{m}$, distributions
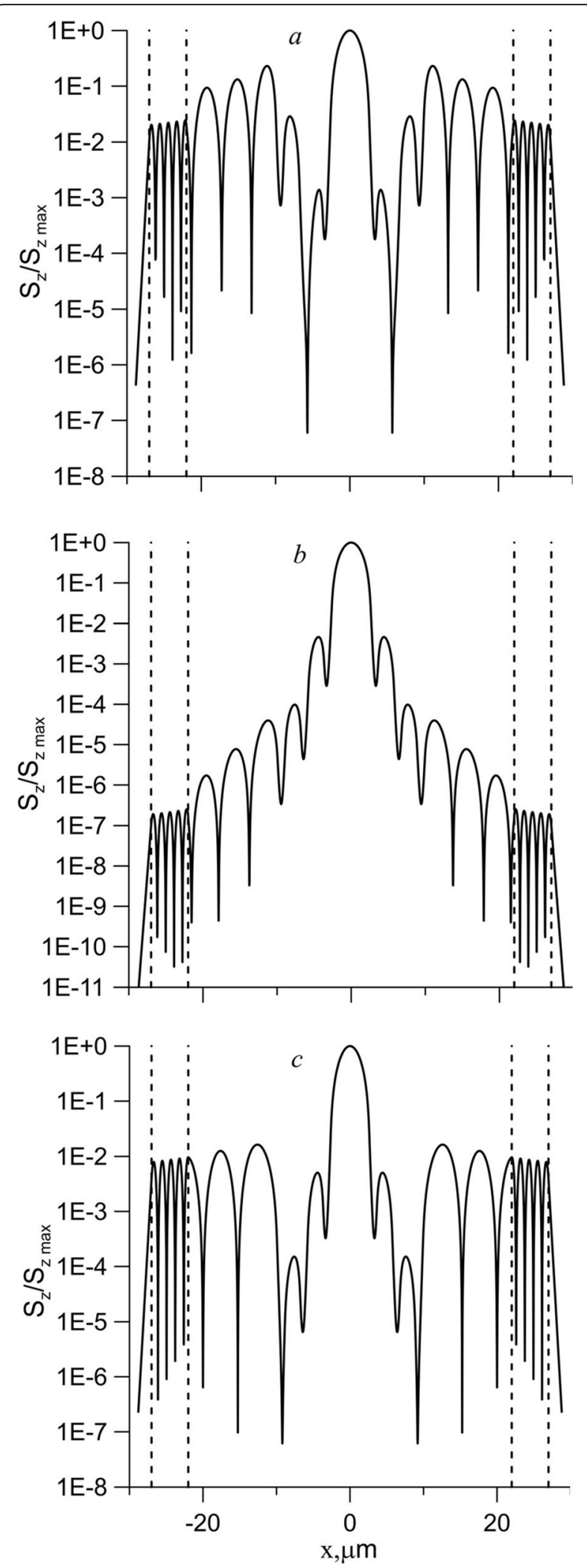

Fig. 6 Distributions of the tapered PCF fundamental mode intensity $S_{z}(x, 0) / S_{z \text { max }}$ at wavelengths $1.244 \mu \mathrm{m}(\mathbf{a}), 1.26 \mu \mathrm{m}(\mathbf{b})$ and $1.279 \mu \mathrm{m}$ (c). Dashed lines indicate polymer coating boundaries 
of the optical density for the PCF mode in the entire spectral range of Fig. 4 visually coincide and look like in Fig. $5 \mathrm{~b}$; more detailed information on the distribution of the mode intensity at different wavelengths is given in Fig. 6, which shows distributions $S_{z}(x, 0) / S_{z \max }$ on a logarithmic scale (the orientation of the coordinate axes $0 x$ and $0 y$ relative to the PCF cross section is clear from Fig. 5).

The plots in Fig. 6a and c are detailed distributions of the mode intensities shown above, in Fig. 5a and b, respectively, and Fig. $6 \mathrm{~b}$ corresponds to the intermediate wavelength between the loss maxima in Fig. 4a. As follows from Fig. 6, the mode loss maxima at wavelengths $1.244 \mu \mathrm{m}$ and $1.279 \mu \mathrm{m}$ in Fig. $4 \mathrm{a}$ are provided by a relatively high normalized intensity of the PCF mode in the absorbing polymer coating. In particular, averaged intensities in Fig. $6 \mathrm{a}$ and $b$, enclosed between dashed lines, are over 4 orders of magnitude higher than the similar intensity in Fig. $6 \mathrm{~b}$.

It should also be noted that the optical field between the PCF core and the absorbing coating in Figs. 5a and 6 has the shape of standing waves, which is natural, since coupling between the PCF core and PCF coating is provided by propagating waves.

Thus, intensity distributions presented in Fig. 6 confirm the connection between the formation of the spectral peaks for the PCF mode losses and the resonant capture of the mode energy by the absorbing coating.

\section{Sensor applications}

Since the modal properties of the PCF absorbing coating can be influenced by the environment, changes in parameters of the environment will lead to a modulation of the leaky modes' propagation constants for the absorbing coating and, due to the coupling of these modes with the PCF mode, to the modulation of the fiber transmission in the vicinity of the resonance wavelengths.

Figures 7 and 8 refer to the adiabatic PCF taper with the polymer coating of thickness $d=5 \mu \mathrm{m}$, surrounded by water, studied in the previous section. They illustrate the use of the LMR corresponding to the direct coupling of the PCF mode with leaky coating modes that take place in the vicinity of $\lambda=1.28 \mu \mathrm{m}$ (see Fig. 4a). Figure 7 shows application of the discussed PCF taper as a refractive index sensor for liquids, such sensors may be used, for example, for bio-chemical sensing [11, 12].

In this case, the sensor efficiency can be characterized by the sensitivity parameter $d \lambda_{\min } / d n_{w}$, where $d \lambda_{\text {min }}$ is the resonance wavelength shift corresponding to an infinitesimal increment in the refractive index of the environment $d n_{w}$, and by a resonance spectral width $\delta \lambda_{0.5}$, which affects the signal-to-noise ratio [13]. The dependence of these parameters on the thickness of the polymer coating is shown in the Table 1 . Here, $\lambda_{\min }$ is the resonance wavelength, Transmission $\left(\lambda_{\min }\right)$ is the corresponding PCF transmission at the initial environment

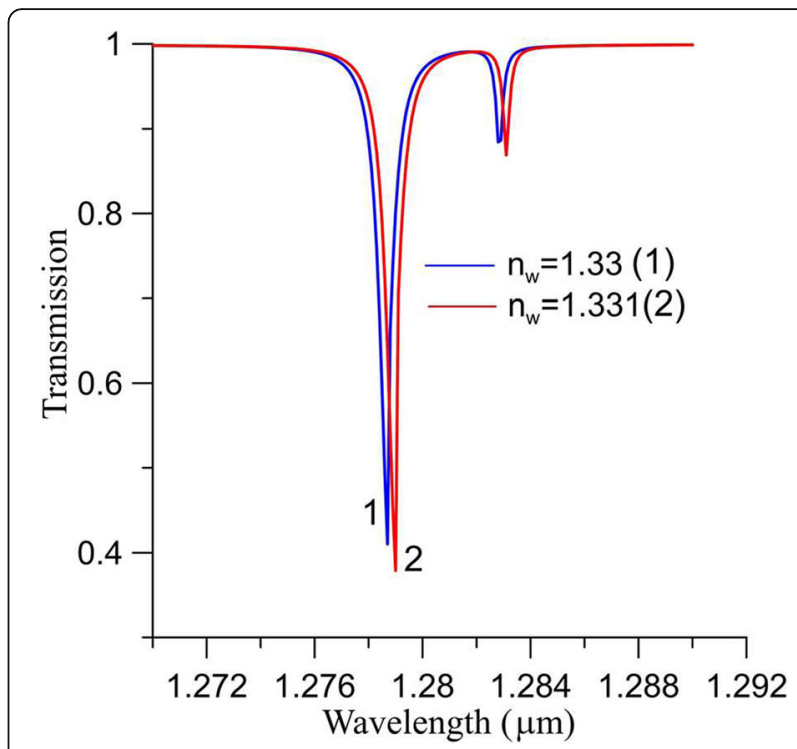

Fig. 7 Shift of the transmission spectrum of the adiabatic taper of the PCF with length $L=3 \mathrm{~cm}$ and a polymer coating of thickness $5 \mu \mathrm{m}$ in dependence on changes of refractive index in the surrounding liquid

refractive index $\mathrm{n}_{\mathrm{w}}=1.33, \delta \lambda_{0.5}$ is the resonance spectral width determined as $\delta \lambda_{0.5}=\lambda_{2}-\lambda_{1}$, where $\lambda_{2}$ and $\lambda_{1}\left(\lambda_{2}>\right.$ $\lambda_{1}$ ) are roots of the equation.

$\operatorname{Transmission}(\lambda)=0.5\left[1+\operatorname{Transmission}\left(\lambda_{\min }\right)\right]$.

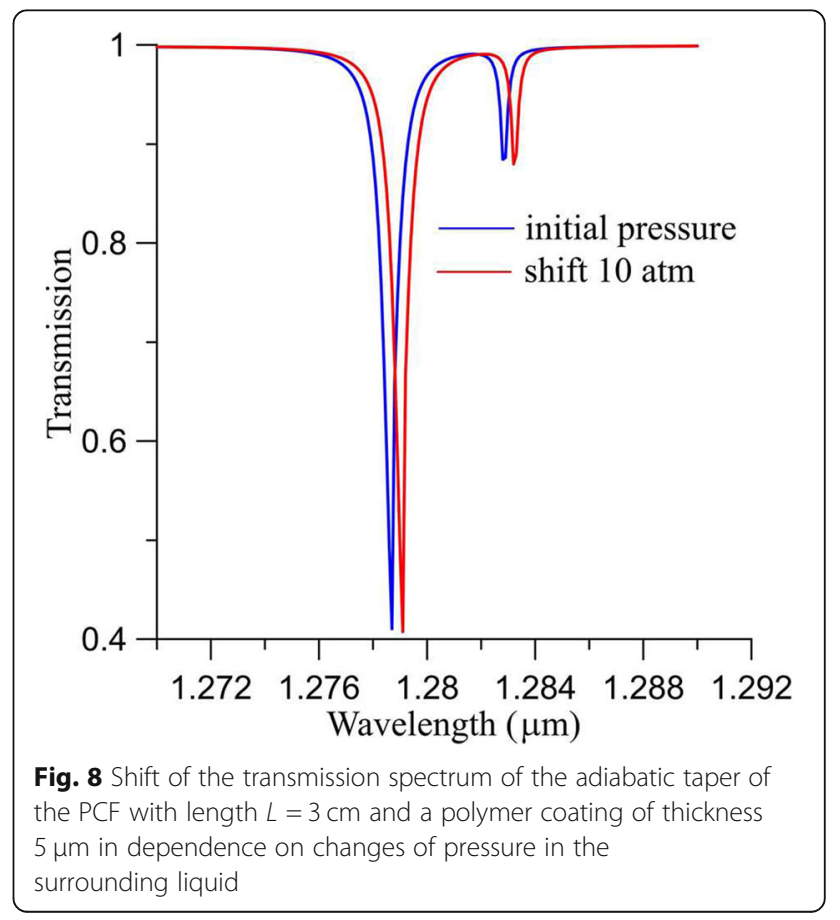


Values of these parameters calculated for different thicknesses of the polymer film are given in the Table 1 .

The tabular data allow us to conclude that in comparison with multimode sensors, in which the absorbing polymer coating is applied directly to the silica fiber core [14], the devices under consideration are less sensitive to changes in the refractive index of the surrounding liquid, but have more than an order of magnitude lower spectral width of resonances; in addition, in contrast to the refractive index sensors studied in [14], the sensitivity of the described PCF sensors decreases as the thickness of the absorbing coating increases. These differences are a consequence of the peculiarities of the interaction of the leaky coating modes with the environment and the single-mode operation of the sensor.

Figure 8 shows the possibility of using the polymer coated adiabatically tapered PCF as a sensor of pressure in a liquid. The liquid pressure sensor may be used, for example, in petroleum pipelines, water conservancy and hydropower applications, hydraulic machinery, etc.

On calculating the pressure sensor, we took into account that the change in pressure results in a change in the coating thickness $\Delta d$ and also in the photo elastic shift $\Delta n_{c}$ of the coating refractive index. Both these factors influence the propagation constants of the coating leaky modes. According to the theory of elasticity [15], the relationship between the increment in a coating thickness and the increment in pressure $\Delta P$ in the environment is given by the formula

$$
\Delta d=-\frac{\Delta P B\left(B^{2}-A_{w}^{2}\right)(1+\sigma)(1-2 \sigma)}{E\left[B^{2}+A_{w}^{2}(1-2 \sigma)\right]},
$$

where $B=A_{w}+d, E$ is Young's modulus, $\sigma$ is Poisson's ratio for the coating. Photo elastic shift $\Delta n_{c}$ was estimated by the formula $\Delta n_{c}=K \Delta P$, where $K$ is Brewster's coefficient. For butyl acrylate, we used $\sigma=0.3, K=10^{-}$ ${ }^{10} 1 / \mathrm{Pa}, E=7 \cdot 10^{8} \mathrm{~Pa}$. Because of the Young's modulus of quartz glass is greater than the last value by two orders, we neglect the slow phase effects caused by PCF cladding and core deformation. In this case, the pressure increments in the surrounding liquid lead to the direct modulation of the PCF transmission. As follows from Fig. 8, the sensitivity of the described pressure sensor

Table 1 Characteristics of the adiabatically tapered PCF with the polymer coating as a refractive index sensor. RIU stands for Refractive Index Unit

\begin{tabular}{lllll}
\hline $\boldsymbol{d}, \boldsymbol{\mu m}$ & $\boldsymbol{\lambda}_{\min , \boldsymbol{\mu m}}$ & Transmission $\left(\lambda_{\min }\right)$ & $\boldsymbol{d} \boldsymbol{\lambda}_{\min } / \boldsymbol{d n _ { \boldsymbol { w } } , \boldsymbol { \mu m } / \mathrm { RIU }}$ & $\delta \lambda_{\mathbf{0 . 5}} \boldsymbol{\mu \mathrm { m }}$ \\
\hline 1.5 & 1.3073 & 0.65 & 0.81 & $5.0 \cdot 10^{-4}$ \\
5 & 1.2787 & 0.41 & 0.31 & $4.5 \cdot 10^{-4}$ \\
10 & 1.2538 & 0.89 & 0.10 & $4.0 \cdot 10^{-4}$ \\
\hline
\end{tabular}

can be estimated as $d \lambda_{\min } / d P=4 \cdot 10^{-5} \mu \mathrm{m} / \mathrm{atm}$. According to Eq. (1), the sensitivity can be changed by varying the thickness of the polymer coating (it will increase with increasing $d$ ).

According to Figs. 7 and 8, sensors of the type described, with the absorbing polymer coating, are well suited to testing parameters in liquids.

Many different absorbing coatings for silica fibers are currently known $[1,2]$, and our calculations have shown that most of such coatings can be used in sensors of the type considered here. However, the described effect of the leaky mode resonance coupling manifests itself in different ways, when low-refractive and high-refractive absorbing coatings are used; this is confirmed by data presented in Figs. 9 and 10. They refer to the coating of tin dioxide doped with antimony (7 wt\%), selectively adsorbing ammonia molecules from air. Calculations are performed for the adiabatic taper with the abovementioned parameters, but surrounded by air instead of water. The $\mathrm{SnO}_{2}$ coating thickness is $0.6 \mu \mathrm{m}$, its complex refractive index $\mathrm{n}_{\mathrm{c}}=1.90819-\mathrm{i} 0.00042$, an adsorption layer of ammonia molecules on the outer surface of the coating has a thickness of $0.37 \mathrm{~nm}$ and a refractive index $n_{a}=1.355$. The parameters of the coating and $\mathrm{NH}_{3}$ molecular layer adsorbed on it are chosen in accordance with the experimental data, obtained using the prism - coupler scheme [16]. Figures 9 and 10 refer to the initial state of the sensor (in the absence of an adsorption layer).

Comparing Figs. 4b and 9b, one can conclude that the transition from the low-refractive coating to the highrefractive coating leads to the significant increase in the attenuation of coating modes at wavelengths corresponding to the intersection points of curves 3 and 4 with curve 2. The resulting stronger mismatch of the complex propagation constants of the PCF core mode and coating modes makes the above coupling - mode model even coarser. This may explain the presence of not two as earlier, but only one loss peak in the vicinity of the dashed curves in Fig. 9a.

Figure 10 compares the PCF mode intensity distributions at the resonant $(\lambda=1.297 \mu \mathrm{m})$ and distant from the resonant $(\lambda=1.26 \mu \mathrm{m})$ (see Fig. 9a) wavelengths. It has a meaning similar to Fig. 6.

An analysis of Fig. 10 allows one to draw conclusions similar to those that were made above when analyzing Fig. 6.

Figure 11 shows the shift of the transmission spectrum for the $\mathrm{SnO}_{2}$ coated adiabatically tapered PCF upon a deposition on the coating of the adsorption layer of ammonia molecules. It is known that the thickness of an adsorption layer of ammonia molecules depends on ammonia concentration in the atmosphere. It is necessary also to remember that ammonia can become a 

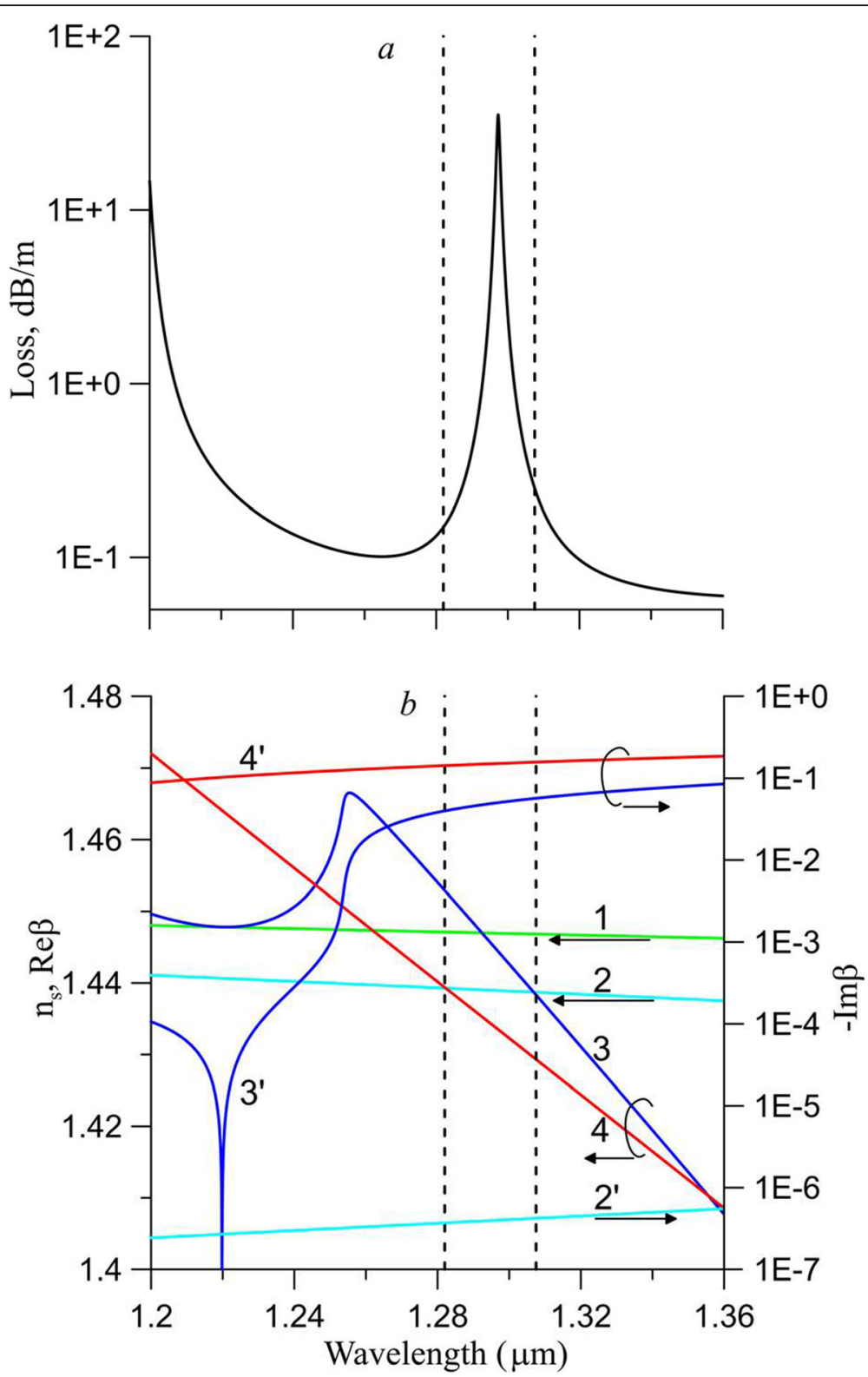

Fig. 9 Spectral dependencies of mode propagation constants for the tapered PCF with the $\mathrm{SnO}_{2}$ coating of thickness $0.6 \mu \mathrm{m}$ surrounded by air; a -loss of the PCF mode, $b$-phase diagram. Curve 1 - quartz glass refractive index, 2 and $2^{\prime}$ - Re $\beta$ and $\operatorname{Im} \beta$ of the PCF core mode, 3 and $3^{\prime}$ - Re $\beta$ and $\operatorname{Im} \beta$ of TE mode of the coating, 4 and $4^{\prime}-\operatorname{Re} \beta$ and $\operatorname{Im} \beta$ of TM mode of the coating. Dashed lines indicate wavelengths of phase synchronism of the PCF core and coating modes

threatening gas that pollutes the atmosphere when too much of it is suddenly exhausted [17].

According to Figs. 9, 10 and 11, sensors of the type described with the high-refractive absorbing coating can be used to detect nanoscale adsorption layers.

\section{Conclusions}

We have theoretically investigated the LMR that was generated by the use of special single-mode PCFs with absorbing thin-film coatings of different thickness and refraction. This LMR has been explained as a result of coupling between fundamental leaky modes of the PCF core and coating. Analysis of intensity distributions for the PCF fundamental mode showed that the interaction of the PCF core mode with the absorbing coating applied to the outer surface of the PCF is carried out not by exponentially decreasing waves, but by waves propagating in the fiber cladding and forming standing waves in the cladding. As a result, the LMR in PCFs can be realized if thin-film coating is applied directly to the outer surface of the PCF cladding without a local modification of the cladding. The LMR results in a multi-peak 

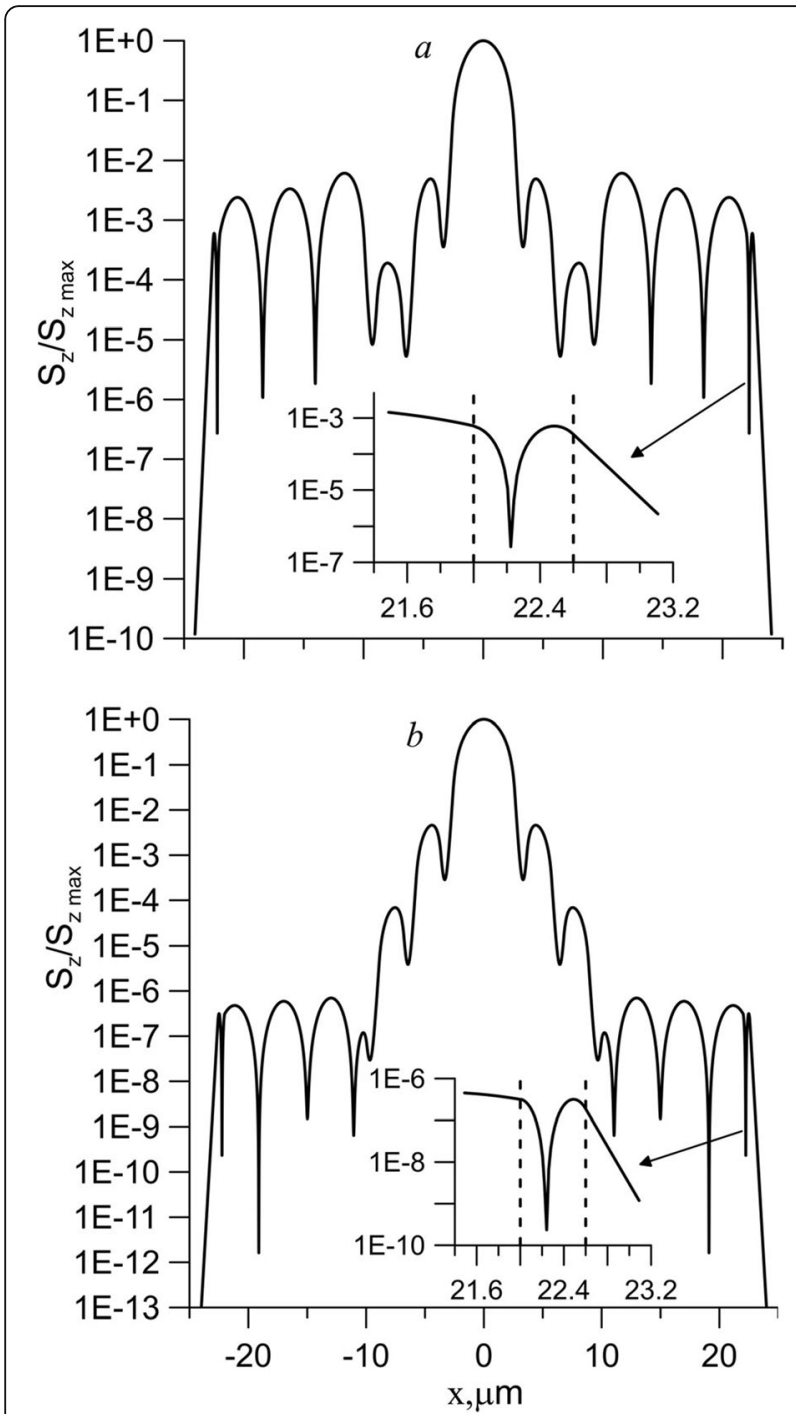

Fig. 10 Distributions of the tapered PCF fundamental mode intensity $S_{z}(x, 0) / S_{z \max }$ at wavelengths $1.297 \mu \mathrm{m}(\mathbf{a})$ and $1.26 \mu \mathrm{m}(\mathbf{b})$ Dashed lines indicate tin dioxide coating boundaries

spectral transmission of the PCF. Two mechanisms for qualitative implementation of such transmission have been proposed. In the first one, the leaky fundamental mode of the PCF core is resonance coupled with leaky modes of the coating. This coupling leads to a resonance capture of the fundamental PCF mode energy by the coating and the release of heat in it. The second mechanism is more complicated and can be observed in PCFs with sufficiently small cladding diameters that can be realized in adiabatically tapered special PCFs. It consists of a resonance coupling of the leaky fundamental PCF mode with cladding modes located between air channels and the absorbing coating. In comparison with the last mechanism, the first one allows narrower spectral absorption bands to be realized; for this reason, it is more attractive for sensor applications. It has been shown, for

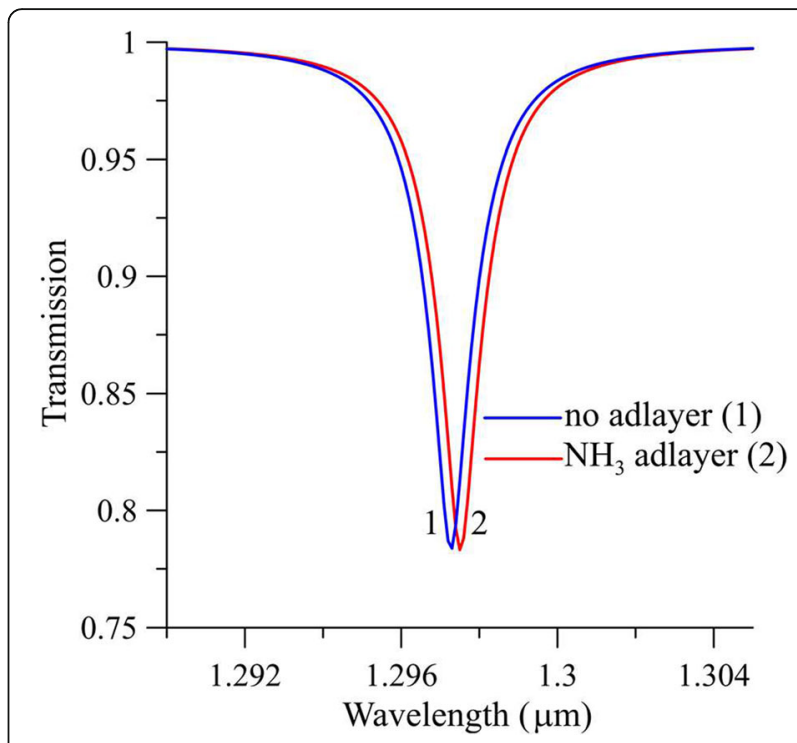

Fig. 11 Shift of the transmission spectrum for the adiabatic taper of the PCF with length $L=3 \mathrm{~cm}$ and tin dioxide coating of thickness $0.6 \mu \mathrm{m}$ at formation of an adsorption layer of ammonia molecules with thickness of $0.37 \mathrm{~nm}$ on coating surface

sensor examples of refractive index, pressure and ammonia molecular adsorption layer, that a small modulation in refractive index and (or) the PCF absorbing coating thickness leads to a detectable modulation of the spectral shift of the PCF transmission in the vicinity of resonance wavelengths. In comparison with known LMR sensors using standard multimode fibers, in which the absorbing coating is applied directly to the fiber core, the investigated sensors are less sensitive to changes in the refractive index of the surrounding liquid, but they have spectral widths of resonance that are more than an order of magnitude lower. The advantages of the sensors considered here are their single-mode operation and the possibility of achieving almost complete elimination of diffraction losses of light energy in the PCF sensitive segment.

In this work, we tried to estimate the fundamental features of the appearance of LMR in PCFs with absorbing coatings, and did not set out to investigate in detail the possibilities of optimizing sensors. The corresponding research and experimental implementation of the considered devices will be presented in a separate publication.

\section{Acknowledgements}

Sotsky A.B., Shilov A.V. and Sotskaya L. I. would like to thank Byelorussian State Scientific Program "Photonics 1.3.03" for partial support of this work. The authors also would like to thank their colleagues Monzón-Hernandez, D., Kir'yanov, A.V., and Calixto S. for productive scientific cooperation.

\section{Authors' contributions}

All authors contributed to the preparation, writing and reviewing of the manuscript. All authors have read and approved the final manuscript. 


\section{Funding}

Declared at acknowledgements.

\section{Availability of data and materials}

Data sharing is not applicable to this article as no datasets were generated.

\section{Declaration}

\section{Competing interests}

The authors declare that they have no competing interests.

\section{Author details}

'Division of Photonics, Centro de Investigaciones en Optica, 37150 León, Mexico. ${ }^{2}$ Mogilev State A. Kuleshov University, 212009 Mogilev, Belarus.

${ }^{3}$ Belarussian-Russian University, 212000 Mogilev, Belarus.

Received: 18 May 2021 Accepted: 25 October 2021

Published online: 22 November 2021

\section{References}

1. Del Villar, I., Arregui, F.J., Zamarreño, C.R., Corres, J.M., Bariain, C., Goicoechea, J., Elosua, C., Hernaez, M., Rivero, P.J., Socorro, A.B., Urrutia, A., Sanchez, P., Zubiate, P., Lopez, D., De Acha, N., Ascorbe, J., Matias, I.R.: Optical sensors based on lossy-mode resonances. Sensors Actuators B Chem. 240, 174-185 (2017). https://doi.org/10.1016/j.snb.2016.08.126

2. Ozcariz, A., Ruiz-Zamarreño, C., Arregui, F.J.: A Comprehensive Review: Materials for the fabrication of optical fiber refractometers based on lossy mode resonance. Sensors. 20(7), 1972 (2020). https://doi.org/10.33 90/s20071972

3. Minkovich, V.P., Kir'yanov, V., Sotsky, A.B., Sotskaya, L.I.: Large-mode-area holey fibers with a few air channels in cladding: modeling and experimental investigation of the modal properties. J. Opt. Soc. Am. B. 21(6), 1161-1169 (2004). https://doi.org/10.1364/JOSAB.21.001161

4. White, T.P., McPhedran, R.C., De Sterke, C.M., Botten, L.C., Steel, M.J.: Confinement losses in microstructured optical fibers. Opt. Lett. 26(21), 1660-1662 (2001). https://doi.org/10.1364/OL.26.001660

5. Sotsky, A.B., Sotskaya, L.I., Minkovich, V.P., Monzón-Hernández, D.: Mode attenuation in microstructured optical fibers with absorbing coating. Tech. Phys. 54(6), 865-873 (2009). https://doi.org/10.1134/\$1063784209060152

6. Malitson, I.H.: Interspecimen comparison of the refractive index of fused silica. J. Opt. Soc. Am. 55(10), 1205-1209 (1965). https://doi.org/10.1364/ JOSA.55.001205

7. Cardenas-Sevilla, G.A., Monzon-Hernandez, D., Minkovich, V.P.: Demonstration of an all-fiber band-rejection filter based on a tapered photonic crystal fiber. Appl. Phys. Express. 5(2), 022502 (2012). https://doi. org/10.1143/APEX.5.022502

8. Minkovich, V.P., Monzón-Hernández, D., Villatoro, J., Sotsky, A.B., Sotskaya, L.I.: Modeling of holey fiber tapers with selective transmission for sensor applications. J. Lightw. Technol. 24(11), 4319-4328 (2006). https://doi.org/1 $0.1109 /$ JLT.2006.884207

9. Sotsky, A.B.: Theory of optical waveguide elements. Mogilev State A, pp. 422-427. Kuleshov University, Mogilev (2011) [in Russian]

10. Sotsky, A.B., Steingart, L.M., Jackson, J.H., Chudakovskii, P.Y., Sotskaya, L.I.: Prism excitation of leaky modes of thin films. Technical Physics. 58(11), 1651-1660 (2013). https://doi.org/10.1134/S106378421311025X

11. Minkovich, V.P., Sotsky, A.B.: Tapered photonic crystal fibers coated with ultra-thin films for highly sensitive bio-chemical sensing. J. Eur. Opt. Soc.Rapid Publ. 15(7), 1-6 (2019). https://doi.org/10.1186/s41476-019-0103-6

12. $\mathrm{Xu}, \mathrm{K}$ : Silicon electro-optic micro-modulator fabricated in standard CMOS technology as components for all silicon monolithic integrated optoelectronic systems. J. Micromech. Microeng. 31(5), 054001 (2021). https://doi.org/10.1088/1361-6439/abf333

13. Sharma, A.K., Gupta, B.D.: On the sensitivity and signal to noise ratio of a step-index fiber optic surface plasmon resonance sensor with bimetallic layers. Opt. Commun. 245(1-6), 159-169 (2005). https://doi.org/10.1016/j. optcom.2004.10.013

14. Del Villar, I., Hernaez, M., Zamarreño, C.R., Sánchez, P., Fernández-Valdivielso, C., Arregui, F.J., Matias, I.R.: Design rules for lossy mode resonance based sensors. Appl. Opt. 51(19), 4298-4307 (2012). https://doi.org/10.1364/AO.51. 004298
15. Landau, L.D., Lifshitz, E.M., Kosevich, A.M., Pitaevskii, L.P.: Theory of Elasticity, Volume 7, Elsevier, 30-39 (1986)

16. Khomchenko, A.V.: Waveguide Spectroscopy of Thin Films, Academic Press, 145-147 (2005)

17. Kim, J., Lee, H., Vo, H.T., Lee, G., Kim, N., Jang, S., Joo, J.B.: Bead-shaped mesoporous alumina adsorbents for adsorption of ammonia. Materials. 13(1375), 1-13 (2020). https://doi.org/10.3390/ma13061375

\section{Publisher's Note}

Springer Nature remains neutral with regard to jurisdictional claims in published maps and institutional affiliations.

\section{Submit your manuscript to a SpringerOpen ${ }^{\circ}$ journal and benefit from:}

- Convenient online submission

- Rigorous peer review

- Open access: articles freely available online

High visibility within the field

- Retaining the copyright to your article

Submit your next manuscript at $\boldsymbol{\nabla}$ springeropen.com 